\title{
Análise da prevalência de erros radiográficos em um serviço de ensino superior do Nordeste do Brasil
}

\author{
Prevalence Analysis of Radiographic Errors in a \\ Service of Graduation Education Institution in the Northeast of Brazil
}

\section{RESUMO}

\author{
Deborah Gondim Lambert Moreira \\ Amanda Katarinny Goes Gonzaga" \\ Laryssa Maria dos Santos Andrade ${ }^{\text {III }}$ \\ Salomão IsRael Monteiro Lourenço Queiroz ${ }^{I V}$ \\ Patricia Teixeira Oliveirav \\ Éricka Janine Dantas da Silveira ${ }^{\mathrm{VI}}$ \\ Ana Miryam Costa de Medeiros ${ }^{\mathrm{VI}}$
}

\begin{abstract}
Objetivo: Determinar a prevalência de erros em radiografias periapicais cometidos pelos acadêmicos de diferentes períodos do curso de Odontologia da Universidade Federal do Rio Grande do Norte, durante o período de um ano. Material e métodos: As radiografias periapicais foram avaliadas por dois examinadores que identificaram e quantificaram os erros observados de acordo com o tipo (técnica e processamento). Para análise estatística, foram utilizados os testes de Kruskal Wallis, Mann-Whitney, Qui-quadrado de Pearson e Exato de Fisher. Resultados: Foram analisadas 522 radiografias periapicais, das quais $236(45,2 \%)$ não apresentaram erros de processamento e/ou técnica e $286(54,8 \%)$ apresentaram pelo menos um erro. Os erros de processamento foram os mais frequentes $(64,3 \%)$, seguidos dos de técnica $(27,2 \%)$. Vinte e quatro radiografias $(8,4 \%)$ apresentaram erros de técnica e processamento. Dos erros de processamento, a presença de ranhuras nas películas radiográficas foi o erro mais comum $(11,9 \%)$. Não foi encontrada associação significativa entre a frequência de erros nas radiografias periapicais e os respectivos períodos. Conclusão: Os erros não diminuíram com o aumento da experiência do operador e a maior parte deles poderia ser evitada se o operador fosse mais atento ao procedimento realizado.

Palavras-chave: Radiografia dentária; Controle de qualidade; Educação em odontologia.
\end{abstract}

\section{ABSTRACT}

Objective: To determine the prevalence of errors in periapical radiographs made by undergraduate students from different periods of the course of Dentistry at Federal University of Rio Grande do Norte during the period of one year. Methods: Periapical radiographs were evaluated by two examiners, which have identified and quantified the observed errors according to type (technical and processing). For statistical analysis was used Kruskal Wallis test, Mann-Whitney, chi-square test and Fisher exact tests. Results: From 522 periapical radiographs analyzed, $236(45.2 \%)$ showed no processing errors and / or technical and $286(54.8 \%)$ had at least one error. Processing errors were the most frequent $(64,3 \%)$, followed by technical errors $(27,2 \%)$. Twenty-four radiographs $(8.4 \%)$ had both processing and technical errors. Of processing errors, the presence of radiographic slots in films was the most common error $(11.9 \%)$. There was no significant association between the frequency of errors in periapical radiographs and the relevant periods. Conclusion: The errors did not decrease with increasing operator experience and most of them could be avoided if the operator was concentrated on the procedure performed.

Keywords: Radiography, dental; Quality control; Education, dental

I Universidade Federal do Rio Grande do Norte (UFRN), Natal/RN - Brasil 


\section{INTRODUÇÃO}

As radiografias periapicais são exames complementares bastante utilizados pelo cirurgião-dentista e possuem uma importância fundamental na clínica odontológica, sendo responsáveis pelo fornecimento de informações complementares para o planejamento, diagnóstico e, consequentemente, tratamento de condições patológicas ${ }^{1}$.

A qualidade da imagem radiográfica é considerada como o resultado da combinação de características de densidade, contraste e nitidez associado ao enquadramento do campo de interesse. Deste modo, é necessário que os protocolos para obtenções das radiografias sejam otimizados, considerando desde o local de armazenamento, o posicionamento do filme e do paciente, e os parâmetros de exposição e de processamento radiográfico. A partir da associação desses fatores, é possível se obter uma radiografia de boa qualidade que contribui para um diagnóstico preciso e planejamento correto do tratamento ${ }^{2}$.

Falhas das técnicas, sejam elas durante a realização da tomada radiográfica ou no processamento dos filmes, resultam em imagens inadequadas e insatisfatórias, que além de gerar interpretações errôneas, acarretam em um aumento da exposição dos pacientes à radiação ionizante. Somados a esses fatores, os erros também envolvem tempo profissional improdutivo, perda de filmes e soluções processadoras, aumentando os custos para clínicas particulares, instituições de ensino e serviços públicos de assistência odontológica ${ }^{3}$.

A análise das principais causas da perda de qualidade nos exames radiográficos, como também a frequência com que elas ocorrem, constitui uma ferramenta fundamental na identificação de falhas no processo ensino-aprendizagem na área da radiologia odontológica $^{2}$. Neste contexto, é importante ressaltar que quando o operador é inexperiente ou está em processo de aprendizagem, os erros são mais frequentes ${ }^{4}$.

Os estudos de investigação de erros radiográficos cometidos por estudantes universitários são importantes, pois estimulam os docentes na busca de melhores métodos e recursos para o processo de ensino, além de proporcionar uma melhoria no cuidado e no armazenamento dessa documentação odontológica, que possui valor legal ${ }^{5}$.

Assim, esta pesquisa objetivou identificar, avaliar e comparar a proporção de erros em radiografias periapicais cometidos pelos acadêmicos do curso de Odontologia da Universidade Federal do Rio Grande do Norte, durante o período de um ano, com o intuito de auxiliar na melhora da qualidade das radiografias obtidas devido a seu valor diagnóstico e legal.

\section{MATERIAL E MÉTODOS}

O presente estudo foi aprovado pelo Comitê de Ética em Pesquisa (CEP) da Universidade Federal do Rio Grande do Norte (UFRN) sob protocolo de numeração 0942632015. Foi realizado um estudo retrospectivo, observacional de avaliação de radiografias periapicais realizadas de janeiro a dezembro do ano de 2014 na Universidade Federal do Rio Grande do Norte.

As exposições radiográficas foram realizadas no aparelho intrabucal GNATUS (Gnatus - Equipamentos Medico Odontológicos LTDA São Paulo, Brasil) e processadas manualmente em câmara escura (labirinto), pelo método tempo-temperatura $\left(2 \mathrm{~min}-4 \mathrm{~min} / 21^{\circ} \mathrm{C}\right)$. Foram avaliadas todas as radiografias intra-orais periapicais, realizadas através da técnica de paralelismo com auxílio de posicionador (Maquira - Indústria de Produtos Odontológicos LTDA - Maringá Paraná, Brasil), provenientes dos prontuários de pacientes adultos do quarto, quinto, sexto, 
sétimo, oitavo e nono períodos. O quarto período corresponde à primeira prática em clínica dos alunos e o nono, à última clínica da graduação.

Foi realizada a padronização do conhecimento de dois examinadores para identificação e quantificação dos erros. Os examinadores analisaram todas as películas individualmente com o auxílio de uma lupa e um negatoscópio de mesa (PROTENI, Araraquara, São Paulo, Brasil). As radiografias foram avaliadas em ambiente sem iluminação artificial ou natural e, após a classificação dos erros, em caso de discordância entre os avaliadores, foi realizada uma nova avaliação até que se chegasse a um consenso. A definição, tipo de erro e sua subdivisão está exemplificada no Quadro 1.

Quadro 1: Classificação dos erros analisados nas radiografias periapicais.

\begin{tabular}{|l|l|}
\hline Categoria dos erros & Subdivisão \\
\hline Erros de técnica & Alongamento \\
\cline { 2 - 2 } & Encurtamento \\
\cline { 2 - 2 } & $\begin{array}{l}\text { Posicionamento da } \\
\text { película de raios-X }\end{array}$ \\
\cline { 2 - 2 } & Presença de meia lua \\
\hline \multirow{4}{*}{$\begin{array}{l}\text { Erros de } \\
\text { processamento da } \\
\text { película de raios-X }\end{array}$} & $\begin{array}{l}\text { Erros no processamento } \\
\text { radiográfico - Fixação e/ } \\
\text { ou revelação }\end{array}$ \\
\cline { 2 - 2 } & $\begin{array}{l}\text { Marcas digitais nas } \\
\text { películas }\end{array}$ \\
\cline { 2 - 2 } & $\begin{array}{l}\text { Películas coladas umas } \\
\text { às outras }\end{array}$ \\
\cline { 2 - 2 } & Ranhuras \\
\hline
\end{tabular}

\section{AnÁlise estatística}

A análise dos dados foi realizada no SPSS versão 22, sendo os dados avaliados de forma descritiva e, posteriormente, realizados testes estatísticos em busca de associações e diferenças significativas. Assumindo a não normalidade e independência da amostra para comparação da quantidade de erros entre as clínicas e suas porcentagens, foram utilizados os testes de Kruskal Wallis e Mann-Whitney, respectivamente. Para verificar associações significativas (nível de significância de 0,05) foram utilizados os testes do Qui-quadrado de Pearson e o Exato de Fisher.

\section{Resultados}

Foram avaliadas 522 radiografias, das quais $286(54,8 \%)$ apresentaram pelo menos um erro de técnica ou processamento. A tabela 1 apresenta a prevalência dos tipos de erros distribuídos de acordo com o período correspondente. De acordo com o total de erros, independente do período, o erro de processamento foi o mais frequente, estando presente em 184 (64,3\%) películas, destacando-se a presença de ranhuras como o tipo de erro de processamento mais comum $(n=62 / 11,9 \%)$. Os erros de técnica estavam presentes em $78(27,2 \%)$ películas. Vinte e quatro $(8,4 \%)$ radiografias apresentaram erros de técnica e de processamento na mesma película. Os erros de processamentos foram mais frequentes em todas as clínicas quando comparados aos de técnica, sendo proporcionalmente mais frequentes na clínica $6(48 / 71,6 \%)$.

Não foi encontrada associação significativa entreoerro(presençaouausência)eosperíodos (p:0,074). Entretanto, o sétimo período, cujos alunos estão em seu antepenúltimo período acadêmico, apresentou proporcionalmente a maior atribuição de erro $(n=58 / 61,7 \%)$. Quando analisada a frequência de cada erro de acordo com o período, observa-se que o erro de técnica do tipo alongamento $(n=5 / 8,9 \%)$ e o de processamento com ranhuras $(n=12 / 21,4 \%)$ estiveram proporcionalmente mais presentes no quinto período. $O$ erro de posicionamento $(n=13 / 13,8 \%)$ apresentou-se mais prevalente no sexto período. O erro de técnica de 
Deborah Gondim Lambert Moreiralet al.

Tabela 1. Avaliação da frequência de erros de acordo com o período da matriz curricular.

\begin{tabular}{|c|c|c|c|c|c|c|c|}
\hline Períodos & & $4^{\circ}$ & $5^{\circ}$ & $6^{\circ}$ & $7^{\circ}$ & $8^{\circ}$ & $9^{\circ}$ \\
\hline & & n (\%) & n (\%) & n (\%) & n (\%) & n (\%) & n (\%) \\
\hline Erros & $\begin{array}{l}\text { Sim } \\
\text { Não }\end{array}$ & \begin{tabular}{|l|}
$7(30,4)$ \\
$16(69,6)$ \\
\end{tabular} & $\begin{array}{l}31(55,4) \\
25(44,6) \\
\end{array}$ & $\begin{array}{l}48(51,6) \\
45(48,4) \\
\end{array}$ & $\begin{array}{l}58(61,7) \\
36(38,3) \\
\end{array}$ & \begin{tabular}{|l|}
$75(51,4)$ \\
$71(48,6)$ \\
\end{tabular} & $\begin{array}{l}67(60,9) \\
43(39,1) \\
\end{array}$ \\
\hline Alongamento & $\begin{array}{l}\text { Sim } \\
\text { Não }\end{array}$ & \begin{tabular}{|l}
$1(4,3)$ \\
$22(95,7)$
\end{tabular} & $\begin{array}{l}5(8,9) \\
51(91,1)\end{array}$ & $\begin{array}{l}5(5,4) \\
88(94,6)\end{array}$ & $\begin{array}{l}7(7,4) \\
87(92,6)\end{array}$ & \begin{tabular}{|l}
$2(1,4)$ \\
$144(98,2)$
\end{tabular} & \begin{tabular}{|l}
$1(0,9)$ \\
$109(99,1)$
\end{tabular} \\
\hline Encurtamento & $\begin{array}{l}\text { Sim } \\
\text { Não }\end{array}$ & \begin{tabular}{|l}
$0(0)$ \\
$23(100)$ \\
\end{tabular} & $\begin{array}{l}1(1,8) \\
55(98,2) \\
\end{array}$ & $\begin{array}{l}0(0) \\
93(100) \\
\end{array}$ & $\begin{array}{l}2(2,1) \\
92(97,9)\end{array}$ & \begin{tabular}{|l|}
$0(0)$ \\
$146(100)$ \\
\end{tabular} & \begin{tabular}{|l|}
$2(1,8)$ \\
$108(98,2)$ \\
\end{tabular} \\
\hline Posicionamento & $\begin{array}{l}\text { Sim } \\
\text { Não } \\
\end{array}$ & \begin{tabular}{|l}
$2(8,7)$ \\
$21(91,3)$ \\
\end{tabular} & \begin{tabular}{|l}
$5(8,9)$ \\
$51(91,1)$ \\
\end{tabular} & \begin{tabular}{|l}
$9(9,7)$ \\
$84(90,3)$ \\
\end{tabular} & $\begin{array}{l}13(13,8) \\
81(86,2) \\
\end{array}$ & \begin{tabular}{|l|}
$13(8,9)$ \\
$133(91,1)$ \\
\end{tabular} & \begin{tabular}{|l|}
$9(8,2)$ \\
$101(91,8)$ \\
\end{tabular} \\
\hline Meia Lua & $\begin{array}{l}\text { Sim } \\
\text { Não }\end{array}$ & \begin{tabular}{|l}
$0(0)$ \\
$23(100)$ \\
\end{tabular} & $\begin{array}{l}2(3,6) \\
54(96,4) \\
\end{array}$ & \begin{tabular}{|l}
$5(5,4)$ \\
$88(94,6)$ \\
\end{tabular} & \begin{tabular}{|l}
$4(4,3)$ \\
$90(95,7)$ \\
\end{tabular} & \begin{tabular}{|l|}
$10(6,8)$ \\
$136(93,2)$ \\
\end{tabular} & \begin{tabular}{|l|}
$8(7,3)$ \\
$102(92,7)$ \\
\end{tabular} \\
\hline $\begin{array}{l}\text { Fixação e/ou } \\
\text { Revelação }\end{array}$ & $\begin{array}{l}\text { Sim } \\
\text { Não }\end{array}$ & \begin{tabular}{|l|}
$2(8,7)$ \\
$21(91,3)$ \\
\end{tabular} & $\begin{array}{l}12(21,4) \\
44(78,6) \\
\end{array}$ & $\begin{array}{l}17(18,3) \\
76(81,7) \\
\end{array}$ & \begin{tabular}{|l}
$22(23,4)$ \\
$72(76,6)$ \\
\end{tabular} & \begin{tabular}{|l|}
$45(30,8)$ \\
$101(69,2)$ \\
\end{tabular} & $\begin{array}{l}27(24,5) \\
83(75,5) \\
\end{array}$ \\
\hline Marcas Digitais & $\begin{array}{l}\text { Sim } \\
\text { Não } \\
\end{array}$ & \begin{tabular}{|l|}
$2(8,7)$ \\
$21(91,3)$ \\
\end{tabular} & \begin{tabular}{|l|}
$2(3,6)$ \\
$54(96,4)$ \\
\end{tabular} & \begin{tabular}{|l|}
$5(5,4)$ \\
$88(94,6)$ \\
\end{tabular} & \begin{tabular}{|l}
$7(7,4)$ \\
$87(92,6)$ \\
\end{tabular} & \begin{tabular}{|l|}
$6(4,1)$ \\
$140(95,9)$ \\
\end{tabular} & $\begin{array}{l}13(11,8) \\
97(88,2) \\
\end{array}$ \\
\hline \multirow[t]{2}{*}{$\begin{array}{l}\text { Películas } \\
\text { Coladas }\end{array}$} & Sim & $0(0)$ & $3(5,4)$ & $2(2,2)$ & $8(8,5)$ & $10(6,8)$ & $8(7,3)$ \\
\hline & Não & $23 / 100$ & $53(94,6)$ & $91(97,8)$ & $86(91,5)$ & $136(93,6)$ & $102(92,7)$ \\
\hline Ranhuras & \begin{tabular}{|l} 
Sim \\
Não
\end{tabular} & \begin{tabular}{|l|}
$3(11)$ \\
$20(87)$ \\
\end{tabular} & $\begin{array}{l}12(21,4) \\
44(78,6) \\
\end{array}$ & \begin{tabular}{|l|}
$11(11,8)$ \\
$82(88,2)$ \\
\end{tabular} & \begin{tabular}{|l}
$8(8,5)$ \\
$86(91,5)$ \\
\end{tabular} & \begin{tabular}{|l|}
$10(6,8)$ \\
$136(93,2)$ \\
\end{tabular} & \begin{tabular}{|l}
$18(16,4)$ \\
$92(83,3)$ \\
\end{tabular} \\
\hline
\end{tabular}

encurtamento $(n=2 / 2,1 \%)$ e processamento com películas coladas $(n=8 / 8,5 \%)$, no sétimo período. Erros de processamento foram os mais encontrados no oitavo período $(n=45 / 30$, $8 \%$ ), enquanto o erro de técnica com presença de meia lua $(n=8 / 7,3 \%)$ e problemas de processamento com marcas digitais nas películas $(13 / 11,8 \%)$ foram mais prevalentes no nono período.

Quando os seis períodos foram categorizados em dois grupos, obtivemos um grupo formado por alunos com menos de $50 \%$ das clínicas concluídas (até o sexto período) e outro grupo formado por alunos com mais de $50 \%$ das clínicas concluídas (a partir do sétimo período). As películas radiográficas dos alunos com mais de $50 \%$ das clínicas concluídas apresentaram uma maior porcentagem de erros ( $n=200 / 57,1 \%$ ), embora essa associação não tenha sido estatisticamente significativa (p:0,123).

Sabendo que uma película radiográfica poderia apresentar mais de um erro, ao avaliar a quantidade de erros por película, foi observada uma média geral de 0,68 $( \pm 0,73)$ de erros, com no máximo quatro erros por película avaliada. Na tabela 2, observa-se a média da quantidade de erros por período.

As radiografias realizadas por alunos do quarto períodoapresentaram menor quantidade de erros por película, no entanto, a comparação entre a proporção de erros por película e os períodos não revelou diferenças significativas $(p=0,179)$. Quando comparada a quantidade de erros por película e a porcentagem de clínicas concluídas (mais ou menos de 50\%) também não foram encontradas diferenças significativas $(p=0,133)$. 
ANÁLISE DA PREVALÊNCIA DE ERROS RADIOGRÁFICOS EM UM SERVIÇO DE ENSINO SUPERIOR DO NORDESTE DO BRASIL

Tabela 2. Quantidade de erros de acordo com o período da matriz curricular

\begin{tabular}{|c|c|c|c|c|c|}
\hline Períodos & $\mathrm{n}$ & Mediana & $Q_{25}-Q_{75}$ & $\begin{array}{ll}\text { MÉDIA } & \text { DOS } \\
\text { POSTOS } & \end{array}$ & $p$ \\
\hline 4 & 23 & 0,0 & $0,0-1,0$ & 207,7 & \multirow[t]{6}{*}{0,179} \\
\hline 5 & 56 & 1,0 & $0,0-1,0$ & 262,9 & \\
\hline 6 & 93 & 1,0 & $0,0-1,0$ & 250,1 & \\
\hline 7 & 94 & 1,0 & $0,0-1,0$ & 277,9 & \\
\hline 8 & 146 & 0,5 & $0,0-1,0$ & 253,7 & \\
\hline 9 & 110 & 1,0 & $0,0-1,0$ & 277,3 & \\
\hline
\end{tabular}

Q25-75: quartis 25 e 75

\section{Dıscussão}

A qualidade da imagem radiográfica é fundamental para o correto diagnóstico e deve ser adquirida com a menor exposição possível do paciente à radiação ionizante. Desta forma, os erros radiográficos devem ser evitados a fim de se realizar o menor número de exposições, e se obter radiografias que permitam o diagnóstico e tratamento precisos ${ }^{6}$. Na literatura, são encontrados vários estudos que avaliaram a qualidade da radiografia periapical realizada por acadêmicos do curso de odontologia 1,2,3,4,5,6,7. Na presente pesquisa, foram avaliadas apenas radiografias periapicais obtidas com o uso de posicionadores, o que teoricamente contribuiria para um menor número de erros, visto que esse dispositivo facilita a execução da técnica radiográfica ${ }^{2}$.

Felippe et al. $^{6}$ avaliaram 1.823 erros em películas radiográficas realizadas por estudantes de graduação e encontraram $33,7 \%$ de erros cometidos quando da utilização do posicionador e $55,4 \%$ quando os alunos não o utilizaram, demonstrando que o uso dos dispositivos diminuiu a incidência de erros em radiografias periapicais ${ }^{6}$. Nesta pesquisa, mesmo com a utilização do posicionador, foi encontrada uma porcentagem significativa de erros $(54,8 \%)$ nas radiografias periapicais analisadas. Isso pode ser explicado pelo fato da maior parte dos erros estarem relacionados ao processamento e não à técnica radiográfica.

Neste estudo, quando somadas as subcategorias dos erros de processamento, este demonstrou maior frequência $(64,3 \%)$, destacando-se a presença de ranhuras como o erro de processamento mais comum $(11,9 \%)$. Em consonância, a pesquisa realizada por Queiroga et al. ${ }^{2}$ também encontrou a presença de ranhuras como o erro mais frequente nas radiografias analisadas $(35,10 \%)^{2}$. Por outro lado, o estudo de Dias et al. ${ }^{3}$ avaliou 1.847 erros radiográficos, dos quais 1.160 estiveram relacionados à técnica, sendo o posicionamento incorreto do filme o mais frequente $(57 \%)^{3}$. Os achados de Carvalho et al. ${ }^{1}$ também evidenciaram maior frequência de erros de técnica. Na presente pesquisa, a quantidade total de erros de técnica foi de apenas $27,2 \%$, o que ressalta que o uso de posicionadores auxiliou o desempenho dos alunos da Faculdade de Odontologia da UFRN durante a execução da radiografia.

Nossos resultados mostraram alta frequência de erros relacionados ao processamento $(64,3 \%)$, o que corrobora os achados do estudo realizado por Fernandes et al. ${ }^{4}$, na Universidade 
Cidade de São Paulo, o qual constataram em um total de 260 radiografias avaliadas, 150 $(57,70 \%)$ apresentando algum tipo de erro radiográfico, sendo os erros de processamento os mais prevalentes. Pesquisadores atribuem os erros de processamento ao fato dos alunos não respeitarem o tempo ideal de imersão da películaradiográfica em solução reveladorae/ou fixadora ${ }^{8}$, o qual deve seguir as recomendações do fabricante e, geralmente, varia de dois a quatro minutos, embora a literatura recomende até o dobro do tempo para que a imagem se torne completamente transparente ${ }^{1}$. Em relação ao tempo de experiência do aluno, não foi encontrada associação significativa entre a frequência de erros nas radiografias periapicais e os respectivos períodos. Outros estudos corroboram estes achados ${ }^{1,3,4,5,6}$, revelando que os erros não diminuem com o aumento da experiência do aluno.

Alguns pesquisadores postularam que a porcentagem de erros radiográficos cometidos por alunos em períodos próximos à conclusão do curso deveria ser reduzida, uma vez que o estudante teria realizado o número derepetições suficientes para fixar a execução da técnica radiográfica corretamente ${ }^{1,7}$. Neste sentido, Mendonça et al. ${ }^{7}$ analisaram um total de 224 radiografias e compararam as diferenças na quantidade de erros radiográficos em relação ao período. Os autores observaram que nos períodos iniciais, o percentual de radiografias insatisfatórias era de $88,2 \%$, passando para $67 \%$ nos períodos finais, o que não corrobora os nossos achados.

De acordo com os resultados obtidos, foi possível também observar que a partir do sétimo período, correspondente a mais de $50 \%$ da carga horária clínica concluída, erros de processamento com presença de películas coladas foram mais frequentes, sendo encontradas associações estatisticamente significativas $(p=0,026)$. Para Silva et al. $^{9}$, a expressiva quantidade de erros de processamento cometidos por alunos em períodos avançados pode ser explicada pelo fato de que ao realizar procedimentos mais especializados, o operador executa a técnica radiográfica rapidamente e isso pode resultar em falhas ocasionadas, principalmente, pela interrupção no processo de fixação, resultando na perda da qualidade radiográfica? ${ }^{\text {. }}$

Um dos fatores limitantes deste estudo foi a ausência de informações sobre a quantidade de repetições realizadas em cada radiografia, sendo difícil inferir se houve exposição desnecessária do paciente à radiação ionizante.

Nos últimos anos, com o surgimento da radiografia digital, a utilização de películas radiográficas e a etapa de processamento químico tornam-se desnecessárias ${ }^{10}$. Além destas vantagens, a radiografia digital fornece uma melhor qualidade de imagem com significativa redução da dose de radiação empregada ${ }^{10}$. Entretanto, o problema como o alto custo dos aparelhos torna limitado o seu uso na prática clínica para cursos de graduação ${ }^{4}$.

Por fim, os resultados deste estudo demonstram que não houve diferença estatisticamente significativa entre o período do operador e a quantidade de erros cometidos, portanto, não se pode provar que os erros diminuem com o aumento da experiência do operador. Esse contrassenso pode ser atribuído à provável falta de atenção dos estudantes durante a realização da técnica radiográfica, visto que eles estão envolvidos com outros procedimentos de maior complexidade.

\section{Conclusões}

Diante do exposto, concluímos que mesmo com menor quantidade de erros observada no quarto período, correspondente aos alunos que iniciam as práticas clínicas sob observação dos docentes, não foi encontrada diferença estatisticamente significativa entre os períodos. Quando avaliada a frequência 
de erros de processamento, observou-se que há uma maior presença de erros a partir do sétimo período do curso. A maioria dos erros encontrados em nosso estudo poderia ser evitada se o operador fosse mais atento ao procedimento realizado.

\section{REFERÊNCIAS}

1. Carvalho PL, Neves ACC, Medeiros JMF, Zõllner NAR, Almeida LD. Erros técnicos nas radiografias intrabucais realizadas por alunos de graduação. Rev Gaúcha Odontol. 2009 Jun; 57(2):151-155.

2. Queiroga MAS, Moreno NPP, Figueiredo CBO, Abreu MHNG, Brasileiro CB. Avaliação dos erros radiográficos cometidos por graduandos de odontologia em técnicas radiográficas intrabucais. Arq Cent Estud Curso Odontol Univ Fed Minas Gerais.2010;46(2):61-65.

3. Dias IM, Furiati PF, Santos EE, Barros CC, Devito $\mathrm{KL}$. Análise de erros radiográficos cometidos por acadêmicos da Faculdade de Odontologia de Juiz de Fora. Arq Cent Estud Curso Odontol Univ Fed Minas Gerais. 2009 Mai; 45(1):51-56.

4. Fernandes AM, Aguiar APS, da Cruz LP, Aivazoglou MU, Gomes APM, da Silva EG, et al. Avaliação dos erros radiográficos cometidos por alunos de graduação durante o tratamento endodôntico. Rev. odontol. Univ. Cid. São Paulo. 2010 Set; 22(3):216-222

5. Lima LR, Lima Júnior, GTDA, Machado Filho JÁ, Freitas SAP. Avaliação da qualidade e do arquivamento de radiografias periapicais na
Clínica de endodontia da Faculdade NOVAFAPI. Odontol. clín.-cient 2010; 9(4):355-358.

6. Felippe MCS, Nassri MRG, Burgos PG, Freitas SFT, Lage-Marques, JL. Quality of periapical radiographs taken by undergraduate students during endodontic treatment. RSBO rev. sulbras. odontol. 2009; 6(1): 63-69.

7. Mendonça DM, de Amorim MEB, Medina PO, Alves Filho AO, de Brito TCCA, Conde NCO. Avaliação de erros em exames radiográficos intrabucais realizados por acadêmicos de odontologia. Rev. Odontol. Univ. Cid. São Paulo 2013; 25(3): 208-15.

8. Lourenço ADA, Pontual ADA, Silveira MMFD, Pontual MLDA. Qualidade da imagem radiográfica quanto ao tempo de leitura em negatoscópio com interrupção da etapa de fixação. Rev. odonto ciênc. 2010 July; 25(1): 78-82.

9. Silva PVRD, Costa RRD, Silva MCD, Iwaki LCV, Takeshita WM. Digital evaluation of the influence of interruption of the fixation process on radiographic contrast and baseplus-fog density in three commercial brands of radiographic films. Rev. Odontol UNESP. 2014 Feb; 43(1): 41-45.

10. Tsesis I, Kamburoglu K, Katz A, Tamse A, Kaffe I, Kfir A. Comparison of digital with conventional radiography in detection of vertical root fractures in endodontically treated maxillary premolars: an ex vivo study. Oral Surg Oral Med Oral Pathol Oral Radiol Endod. 2008; 106(1): 124-8.

Submetido em: 26-6-2016

Aceito em: 18-8-2016 